\title{
Comparison of Metabolic Network between Muscle and Intramuscular Adipose Tissues in Hanwoo Beef Cattle Using a Systems Biology Approach
}

\author{
Hyun-Jeong Lee, ${ }^{1}$ Hye-Sun Park, ${ }^{2}$ Woonsu Kim, ${ }^{2}$ Duhak Yoon, ${ }^{3}$ and Seongwon Seo ${ }^{2}$ \\ ${ }^{1}$ Division of Animal Genomics and Bioinformatics, National Institute of Animal Science, Rural Development Administration, \\ Suwon 441-706, Republic of Korea \\ ${ }^{2}$ Department of Animal Biosystem Sciences, Chungnam National University, 99 Daehak-ro, Yuseong-gu, \\ Daejeon 305-764, Republic of Korea \\ ${ }^{3}$ Department of Animal Science, Kyungpook National University, Sangju 742-170, Republic of Korea
}

Correspondence should be addressed to Seongwon Seo; swseo@cnu.kr

Received 21 July 2014; Revised 7 October 2014; Accepted 30 October 2014; Published 13 November 2014

Academic Editor: Graziano Pesole

Copyright () 2014 Hyun-Jeong Lee et al. This is an open access article distributed under the Creative Commons Attribution License, which permits unrestricted use, distribution, and reproduction in any medium, provided the original work is properly cited.

The interrelationship between muscle and adipose tissues plays a major role in determining the quality of carcass traits. The objective of this study was to compare metabolic differences between muscle and intramuscular adipose (IMA) tissues in the longissimus dorsi (LD) of Hanwoo (Bos taurus coreanae) using the RNA-seq technology and a systems biology approach. The LD sections between the 6 th and 7th ribs were removed from nine (each of three cows, steers, and bulls) Hanwoo beef cattle (carcass weight of $430.2 \pm$ $40.66 \mathrm{~kg}$ ) immediately after slaughter. The total mRNA from muscle, IMA, and subcutaneous adipose and omental adipose tissues were isolated and sequenced. The reads that passed quality control were mapped onto the bovine reference genome (build bosTau6), and differentially expressed genes across tissues were identified. The KEGG pathway enrichment tests revealed the opposite direction of metabolic regulation between muscle and IMA. Metabolic gene network analysis clearly indicated that oxidative metabolism was upregulated in muscle and downregulated in IMA. Interestingly, pathways for regulating cell adhesion, structure, and integrity and chemokine signaling pathway were upregulated in IMA and downregulated in muscle. It is thus inferred that IMA may play an important role in the regulation of development and structure of the LD tissues and muscle/adipose communication.

\section{Introduction}

Hanwoo, the dominant breed for beef production in Korea, is known for yielding high quality beef with intense marbling and a high percentage of unsaturated oleic fatty acid [1]. The marbling score is determined by the degree of intramuscular adipose (IMA) deposition in the exposed longissimus dorsi (LD) muscle tissue at the 13th rib interface. Consequently, understanding the interrelationship between muscle and adipose tissues in LD is important for improving carcass traits in Hanwoo beef cattle.

Muscle and adipose tissues exhibit distinct metabolism: energy expenditure occurs mainly in muscle while adipose is the main energy storage tissue. Even within adipose tissues, metabolic activities and their regulation are differed by their anatomical locations (e.g., IMA, subcutaneous adipose
(SUA), and omental adipose (OMA) depots) [2]. For instance, there are biological differences between SUA and IMA tissues in terms of development, lipid biosynthesis, and substrate preference [3]. In addition, there has been accumulated scientific evidence that adipose and muscle tissues communicate and coregulate each other by secreting various endocrine signal molecules [4-6]. It is reported that the adipose tissue plays a critical role in insulin sensitivity, substrate selection, and oxidative metabolism of skeletal muscle $[7,8]$. Little, however, has been studied on differences in metabolic gene network between muscle and IMA in an aspect of deciphering cross communication between these tissues in the LD of beef cattle.

RNA-seq is a powerful tool for profiling and quantifying transcriptome using deep-sequencing technologies [9]. Transcriptome analysis using RNA-seq provides biological insight 
to understand underlying molecular basis of metabolic differences among different tissues. Recently, the transcriptome expression profile of muscle $[10,11]$ or adipose $[12,13]$ tissues in cattle was identified using the RNA-seq technology, and Lee et al. [13] have demonstrated the different depot-specific adipogenesis in Hanwoo. To the best our knowledge, no research has been reported on comparing gene expression profile between muscle and IMA in the LD of beef cattle using RNA-seq.

The objective of this study is thus to compare metabolic differences between muscle and IMA tissues in the LD of Hanwoo on the basis of expression of metabolic gene network using RNA-seq technology and a systems biology approach.

\section{Materials and Methods}

Animal use, care, and experimental protocols for this experiment were reviewed and preapproved by the Institutional Animal Care and Use Committee of the National Institute of Animal Science (number 2010-042).

2.1. Animals and Sample Preparation. A total of nine (3 cows, 3 steers, and 3 bulls) Hanwoo cattle (Bos taurus coreanae) were used in this study. They were fed the same diet and managed at the same location, Hanwoo Experimental Station in National Institute of Animal Science, throughout the experiment. The average ( \pm standard deviation) carcass weight of the cattle at slaughter was $430.2( \pm 40.66) \mathrm{kg}$.

Immediately after slaughter, the LD sections between the 6 th and 7 th ribs were removed, and the muscle, IMA, and SUA tissues were separated and sampled from this portion. The OMA tissue was taken within the lesser curvature of the abomasum. All of the tissue samples were immediately frozen using liquid nitrogen and stored at $-80^{\circ} \mathrm{C}$ until the analysis.

2.2. Total RNA Extraction and RNA-seq Analysis. Total RNA of muscle, IMA, SUA, and OMA tissues were isolated using TRIzol (Invitrogen) and an RNeasy RNA purification kit with DNase treatment (Qiagen). The mRNA was isolated from the total RNA using oligo-dT beads and was reverse transcribed into double strand cDNA fragments. Constructing and sequencing RNA-seq library for each sample were carried out based on protocols of Illumina HiSeq2000 to generate 101 pair-end reads. Quality of RNA-seq reads from all of the tissues was checked using FastQC. The reads that passed the quality control were mapped to Bovine Taurus genome (bosTau6) from UCSC using Tophat2 (v2.0.2) and were counted using HTseq (v0.5.3p3). The gene models and annotations of the bovine genome were obtained from the Ensembl release 72 [14]. The RNA sequencing data from this study have been submitted to the NCBI Gene Expression Omnibus (GEO) under the accession number of GSE39618 (http://www.ncbi.nlm.nih.gov/geo/).

2.3. Statistical and Network Analysis. Using the DESeq package in $R$ and Bioconductor differentially expressed genes (DEG) across various tissues were identified by analyzing the mapped RNA sequencing reads [15]. In the DEG analysis a negative binomial model was used for normalization, and a generalized linear model was fitted with sex of the cattle as a block in order to account for the variations from the differences in sex. The Bonferroni test with an experimentalwise alpha level of 0.05 was used to identify the list of genes differentially expressed among the tissues. The hierarchical clustering and principle component analysis clustering analyses on DEG were conducted and visualized using the DESeq package [15].

Among the DEG across the tissues, tissue-specific oppositely regulated genes (ORG) were identified by pairwise comparisons of the expression of each gene among different tissues. The criterion used in this study was the sign of $\log 2$ of the fold change which indicates a relative level of expression of each gene between two tissues. If the sign of log2 of the fold change of a gene was different in a tissue compared to the other three tissues, the gene was regarded as a tissue-specific ORG. Consequently muscle-specific up- or downregulated genes were relatively less or more expressed, respectively, in all of the adipose tissues. Likewise, intramuscular adiposespecific up- or downregulated genes were relatively less or more expressed, respectively, in muscle and the other adipose tissues.

Functional annotations and enrichment tests of tissuespecific ORG were carried out using the Database for Annotation, Visualization and Integrated Discovery (DAVID) [16]. The significantly enriched KEGG pathways in each of the four different gene sets (upregulated in IMA, downregulated in IMA, upregulated in MUS, and downregulated in MUS) were separately identified against the annotated bovine genome using the Bonferroni test at $P<0.05$.

\section{Results}

3.1. Identification of Oppositely Regulated Genes (ORG). Totals of $34.2,35.8,35.1$, and $38.1 \mathrm{Mb}$ of raw reads were obtained on average from muscle, IMA, SUA, and OMA tissues, respectively. More than $99.5 \%$ of the reads were remained after the quality filtering passed through the quality control, and more than $95.9 \%$ of these were mapped to the reference genome (Supplementary Table 1; see Supplementary Material available online at http://dx.doi.org/10.1155/ 2014/679437).

The DEG analysis identified that a total of 7,282 genes were differentially expressed among muscle, IMA, SUA, and OMA tissues. The hierarchical clustering analysis on the expressions of DEG clearly showed distinct patterns of gene expression between muscle and adipose tissues and a unique gene expression profile of IMA compared to OMA and SUA (Figure 1(a)). This was also confirmed by a clustering analysis using principle component analysis (Figure 1(b)). Regardless of sexes, SUA and OMA were clustered together, and there were the other two distinct clusters: muscle and IMA.

The total number of muscle tissue-specific ORG was 6,385 genes in which 2,803 genes were upregulated while 3,582 genes were downregulated. On the other hand, a relatively fewer number of genes $(3,113)$ were differentially expressed in intramuscular adipose tissue: 1,970 genes were upregulated while 1,143 were downregulated. 


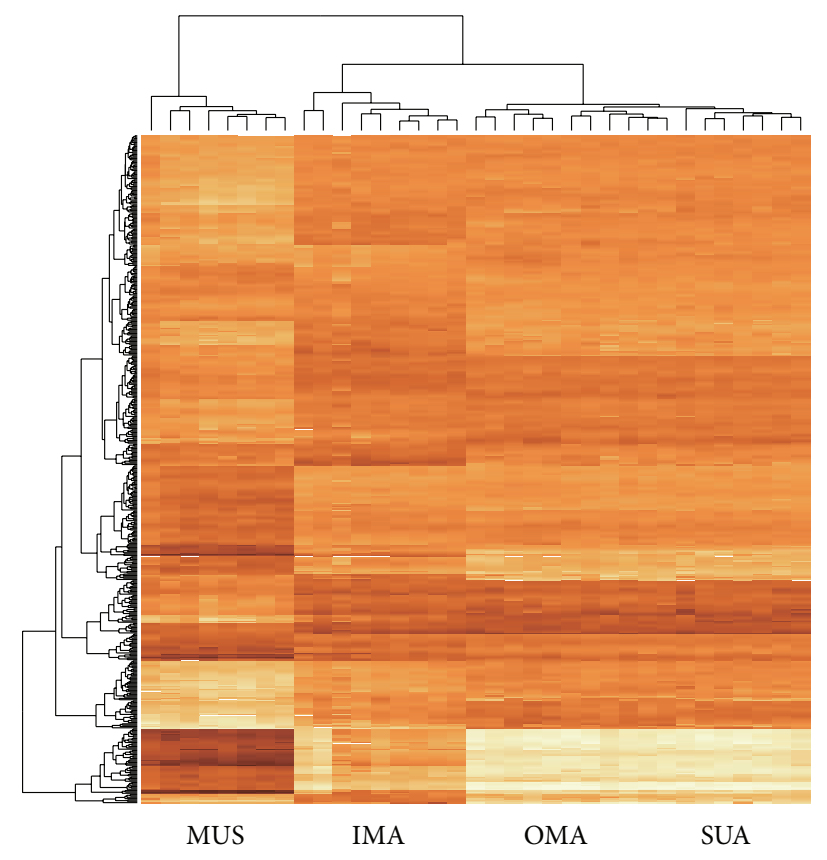

(a)

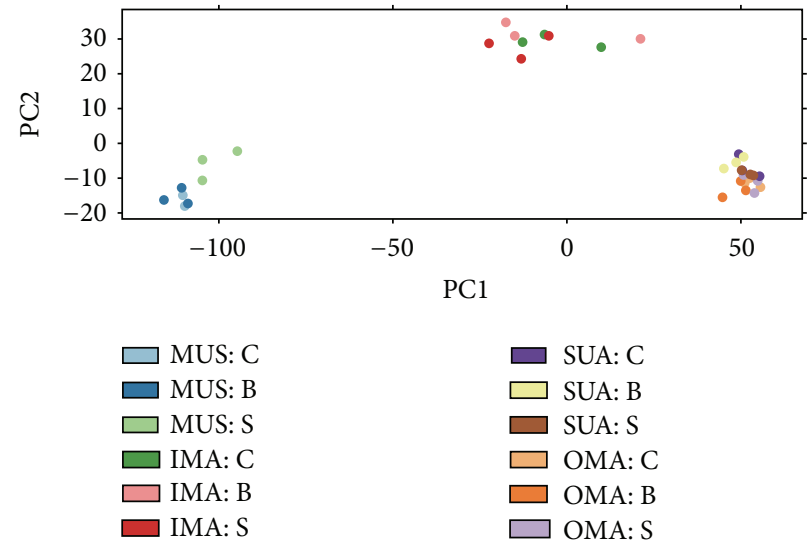

(b)

FIGURE 1: Transcriptomic profiles and clustering of differentially expressed genes (DEG) among four different tissues and three sexes in Hanwoo. The tissues were muscle (MUS), intramuscular adipose (IMA), subcutaneous adipose (SUA), and omental adipose (OMA), and the sexes were cow (C), bull (B), and steer (S). (a) Heat map of levels of gene expressions and hierarchical clustering and (b) clustering using principle component analysis of DEG.

The KEGG pathway enrichment analysis on each of the four sets of genes (upregulated in IMA, downregulated in IMA, upregulated in MUS, and downregulated in MUS) revealed the opposite direction of metabolic regulation between MUS and IMA (Tables 1 and 2). Among nine significantly upregulated KEGG pathways in the IMA, five pathways (i.e., focal adhesion, ECM-receptor interaction, axon guidance, chemokine signaling pathway, and regulation of actin cytoskeleton) were downregulated in the muscle tissue. On the other hand, among 11 downregulated pathways in the IMA, seven pathways (i.e., oxidative phosphorylation Parkinson's disease, Huntington's disease, Alzheimer's disease, citric acid cycle, proteasome, and pyruvate metabolism) were upregulated in muscle (Table 1). In addition, valine, leucine, and isoleucine degradation, propanoate metabolism, fatty acid degradation, and butanoate metabolism were downregulated in the intramuscular adipose tissue. Seven out of 13 upregulated pathways in the muscle tissue were downregulated in the intramuscular adipose tissues, while five out of 12 downregulated pathways in muscle were upregulated in intramuscular adipose (Table 2). Interestingly, it was indicated that the KEGG pathway of dilated cardiomyopathy was upregulated in both IMA and MUS although different ORG was identified.

Metabolic gene network analysis clearly indicated that oxidative metabolism, such as oxidative phosphorylation, citric acid cycle, pyruvate metabolism, glycolysis, and purine metabolism, was upregulated in the muscle tissue but downregulated in the intramuscular tissue. Oxidation of branch-chained amino acids and short-chain fatty acids was specifically downregulated in the intramuscular tissue compared to muscle and subcutaneous adipose and omental adipose tissues.

Interestingly enough, pathways involved in regulation of cell adhesion, structure, and integrity (i.e., ECM adhesion, ECM-receptor interaction, axon guidance, and regulation of actin cytoskeleton) and chemokine signaling pathway were upregulated in the intramuscular adipose tissue and downregulated in the muscle tissue. It is thus speculated that intramuscular adipose tissue may play an important role in regulation of development and structure of the LD tissues and muscle and adipose communication.

\section{Discussion}

Carcass traits are influenced by various factors including genotype, age, nutrition, and management. The IMA and back fat thickness are increased by age [17] and time on feed [18]. The back fat thickness increases more rapidly than marbling score by time on feed. Therefore, extending the feeding period to improve carcass traits may be counterproductive [18]. The IMA develops later on the course of growing and behaves differently from other types of adipose (e.g., subcutaneous and omental adipose tissues) in terms of cellularity and metabolic characteristics [2], and it secretes signals and cross talks with the muscle tissue [4]. Thus, we need to find a way to increase the intramuscular adipose content without deposition of other types of adipose tissues. Better understanding of the interrelationship between intramuscular adipose and muscle tissues in LD is a prerequisite. 
TABLE 1: List of oppositely up- or downregulated KEGG pathways in the longissimus dorsi intramuscular adipose tissue compared to muscle and subcutaneous adipose and omental adipose tissues in Hanwoo.

\begin{tabular}{|c|c|c|c|c|}
\hline & KEGG ID & Pathway & Fold enrichment & Bonferroni \\
\hline \multirow{9}{*}{ Upregulated } & bta04510 & Focal adhesion $^{\dagger}$ & 2.7 & $7.0 E-09$ \\
\hline & bta04512 & ECM-receptor interaction $^{\dagger}$ & 3.7 & $8.2 E-08$ \\
\hline & bta04360 & Axon guidance ${ }^{\dagger}$ & 2.8 & $1.5 E-05$ \\
\hline & bta05200 & Pathways in cancer & 1.8 & $1.6 E-03$ \\
\hline & bta04270 & Vascular smooth muscle contraction & 2.4 & $3.7 E-03$ \\
\hline & bta04062 & Chemokine signaling pathway ${ }^{\dagger}$ & 2.0 & $9.9 E-03$ \\
\hline & bta05412 & Arrhythmogenic right ventricular cardiomyopathy & 2.8 & $1.3 E-02$ \\
\hline & bta04810 & Regulation of actin cytoskeleton ${ }^{\dagger}$ & 1.9 & $1.9 E-02$ \\
\hline & bta05414 & Dilated cardiomyopathy & 2.5 & $4.2 E-02$ \\
\hline \multirow{11}{*}{ Downregulated } & bta00190 & Oxidative phosphorylation $^{\ddagger}$ & 6.2 & $2.4 E-23$ \\
\hline & bta05012 & Parkinson’s disease $\mathrm{e}^{\ddagger}$ & 6.0 & $7.9 E-21$ \\
\hline & bta05016 & Huntington's disease $\mathrm{e}^{\ddagger}$ & 4.8 & $7.5 E-18$ \\
\hline & bta05010 & Alzheimer's disease $\mathrm{e}^{\ddagger}$ & 4.8 & $8.4 E-17$ \\
\hline & bta00020 & Citrate cycle (TCA cycle) $)^{\ddagger}$ & 10.5 & $4.9 E-13$ \\
\hline & bta 03050 & Proteasome $e^{\ddagger}$ & 7.0 & $1.4 E-08$ \\
\hline & bta00280 & Valine, leucine, and isoleucine degradation & 5.9 & $1.2 E-05$ \\
\hline & bta00640 & Propanoate metabolism & 6.7 & $8.8 E-05$ \\
\hline & bta00071 & Fatty acid degradation & 5.4 & $8.7 E-04$ \\
\hline & bta00650 & Butanoate metabolism & 5.4 & $8.1 E-03$ \\
\hline & bta00620 & Pyruvate metabolism ${ }^{\ddagger}$ & 4.7 & $2.7 E-02$ \\
\hline
\end{tabular}

${ }^{\dagger}$ Downregulated in the muscle tissue.

${ }^{\ddagger}$ Upregulated in the muscle tissue.

The present study identified a large number $(7,281)$ of DEG among muscle, IMA, SUA, and OMA tissues in Hanwoo using RNA-seq. The DEG obtained using the RNA-seq analysis were not fully cross validated by qRT-PCR in this study although some of the genes showed a significant and strong correlation between RNA-seq and qRT-PCR previously. In our previous analysis with the same RNA samples, the expression levels of ten randomly selected genes each from IMA, SUA, and OMA are higher than 0.85 of Pearson's correlation coefficient [13].

Clustering analysis of DEG showed that each tissue exhibited unique gene expression pattern regardless of genders (i.e., cow, steer, or bull) or biological replications even though gene expression profiles of SUA and OMA tissues were similar. On the basis of different gene expression pattern between muscle and IMA, it is clear that IMA was successfully isolated from LD in this study in spite of its experimental difficulties. Relative to muscle, the adipose tissues clustered together, and IMA showed different pattern from OMA and SUA tissues. Sheng et al. [12] reported a total of 953, 1,534, and 2,026 DEG between IMA and SUA, IMA and perirenal adipose, and SUA and perirenal adipose tissues, respectively, and found 323 DEG in IMA compared to external adipose tissues. Transcriptome studies in other species have also reported that gene expression patterns were clustered by the tissue rather than sex or breed types [19-21].

We identified not only differentially expressed but also muscle- or intramuscular adipose-specific oppositely (upversus down)regulated genes. A relatively larger number of genes were oppositely regulated in the muscle tissue compared to the others. Metabolic pathway enrichment analysis with the ORG in muscle and IMA revealed the opposite direction of metabolic regulation between muscle and IMA: oxidative pathways were upregulated in muscle but downregulated in intramuscular adipose. Pyruvate metabolism, citric acid cycle, and oxidative phosphorylation were upregulated in muscle. Particularly the genes encoding two core enzymes of pyruvate metabolism, lactate dehydrogenase, and pyruvate dehydrogenase complex were oppositely upregulated in muscle compared to adipose tissues. Pyruvate metabolism is known to play a pivotal role in aerobic and anaerobic energy metabolism. When oxygen is sufficient, pyruvate is oxidized to acetyl-CoA by pyruvate dehydrogenase. Acetyl-CoA then enters into citric acid cycle, and oxidative phosphorylation generates ATP. On the other hand, during intense muscular activity, pyruvate is converted to lactate by lactate dehydrogenase anaerobically $[22,23]$.

The pathways involved in regulation of cell adhesion, structure, and integrity and chemokine signaling pathway were upregulated in intramuscular adipose but downregulated in muscle. Specifically ECM adhesion and ECMreceptor interaction pathways are critical in these functions. These pathways have also been proposed to play an important role in the intramuscular fat deposition in chicken [24]. The ECM surrounds cells and accomplishes a number of specific functions, such as cell adhesion, migration, proliferation, and differentiation. In muscle, cells are tightly bound together, and the extracellular spaces containing the ECM are limited. 
TABLE 2: List of oppositely up- or downregulated KEGG pathways in the longissimus dorsi muscle tissue compared to intramuscular, subcutaneous, and omental adipose tissues in Hanwoo.

\begin{tabular}{|c|c|c|c|c|}
\hline & KEGG ID & Pathway & Fold enrichment & Bonferroni \\
\hline \multirow{13}{*}{ Upregulated } & bta05012 & Parkinson's disease $^{\dagger}$ & 4.2 & $1.4 E-27$ \\
\hline & bta05010 & Alzheimer's disease ${ }^{\dagger}$ & 3.7 & $5.3 E-26$ \\
\hline & bta00190 & Oxidative phosphorylation $^{\dagger}$ & 4.0 & $8.3 E-26$ \\
\hline & bta05016 & Huntington's disease $^{\dagger}$ & 3.4 & $2.2 E-22$ \\
\hline & bta 03050 & Proteasome ${ }^{\dagger}$ & 5.3 & $9.5 E-15$ \\
\hline & bta04260 & Cardiac muscle contraction & 3.7 & $3.2 E-10$ \\
\hline & bta00020 & Citrate cycle (TCA cycle) ${ }^{\dagger}$ & 5.1 & $8.7 E-09$ \\
\hline & bta 05410 & Hypertrophic cardiomyopathy (HCM) & 2.7 & $4.9 E-04$ \\
\hline & bta05414 & Dilated cardiomyopathy & 2.5 & $1.9 E-03$ \\
\hline & bta00230 & Purine metabolism & 2.0 & $2.8 E-03$ \\
\hline & bta04120 & Ubiquitin mediated proteolysis & 2.1 & $3.2 E-03$ \\
\hline & bta00620 & Pyruvate metabolism ${ }^{\dagger}$ & 3.0 & $2.7 E-02$ \\
\hline & bta00010 & Glycolysis/gluconeogenesis & 2.6 & $2.9 E-02$ \\
\hline \multirow{12}{*}{ Downregulated } & bta04510 & Focal adhesion ${ }^{\ddagger}$ & 1.9 & $1.3 E-06$ \\
\hline & bta 04512 & ECM-receptor interaction ${ }^{\ddagger}$ & 2.4 & $5.0 E-06$ \\
\hline & bta04640 & Hematopoietic cell lineage & 2.3 & $1.9 E-05$ \\
\hline & bta04062 & Chemokine signaling pathway $^{*}$ & 1.8 & $4.7 E-05$ \\
\hline & bta04360 & Axon guidance ${ }^{\ddagger}$ & 2.0 & $7.5 E-05$ \\
\hline & bta04670 & Leukocyte transendothelial migration & 2.0 & $1.1 E-04$ \\
\hline & bta04060 & Cytokine-cytokine receptor interaction & 1.7 & $6.3 E-04$ \\
\hline & bta00600 & Sphingolipid metabolism & 2.7 & $3.7 E-03$ \\
\hline & bta 04514 & Cell adhesion molecules (CAMs) & 1.8 & $4.2 E-03$ \\
\hline & bta04810 & Regulation of actin cytoskeleton $^{*}$ & 1.6 & $5.9 E-03$ \\
\hline & bta04142 & Lysosome & 1.8 & $6.6 E-03$ \\
\hline & bta04666 & Fc gamma R-mediated phagocytosis & 2.0 & $8.6 E-03$ \\
\hline
\end{tabular}

${ }^{\dagger}$ Downregulated in the intramuscular adipose tissue.

${ }^{\ddagger}$ Upregulated in the intramuscular adipose tissue.

The ECM is mainly composed of two macromolecules: glycosaminoglycans and fibrous proteins. Glycosaminoglycans are usually covalently linked to core proteins to form proteoglycans, and fibrous proteins can be divided into two functional groups: structural (e.g., collagen, elastin) and adhesive (e.g., fibronectin, laminin, and vitronectin) types [25]. Transmembrane molecules such as integrins mediate interactions between cells and the ECM. In our study, collagen, integrin, laminin, fibronectin, and other cell junction related genes were significantly upregulated in IMA. Interestingly enough, some of the upregulated pathways in the IMA tissue were highly expressed in the LD of cattle embryo. He and Liu [11] identified DEG in the LD muscle between embryo and 30month-old adult cattle using RNA-seq and conducted KEGG pathway enrichment analysis. They reported that KEGG pathways of axon guidance, hypertrophic cardiomyopathy, dilated cardiomyopathy, pathways in cancer, and regulation of actin cytoskeleton were significantly and highly expressed in embryo compared to adult.

To the best of our knowledge, this study is the first scientific report suggesting that intramuscular adipose tissue may play an important role in communication with muscle and regulation of development and structural integrity of the longissimus dorsi tissue. The findings from this study will provide a scientific ground for finding better strategies to improve carcass traits of beef cattle.

Moreover, this study used the RNA-seq technology and a systems biology approach, which are relatively new to our research field but powerful tools for quantifying and profiling transcriptome (the whole set of expressed genes) and deciphering underlying molecular basis of metabolic differences among different tissues. Application of these recent techniques in the animal science field should be very helpful for expanding our understanding of animal biology.

\section{Conflict of Interests}

The authors declare that there is no conflict of interests regarding the publication of this paper.

\section{Acknowledgment}

This research was supported by a grant from the NextGeneration BioGreen 21 Program (no. PJ00819107), Rural Development Administration, Republic of Korea. 


\section{References}

[1] K. K. Jung and C. B. Choi, "Development of technologies to improve competitiveness of Hanwoo," in S. Report to the Ministry of Agriculture, pp. 85-98, Seoul, Korea, Ministry of Ariculture, Food and Rural Affairs, 2003.

[2] A. W. B. Joe, Y. Lin, Y. Even, A. W. Vogl, and F. M. V. Rossi, "Depot-specific differences in adipogenic progenitor abundance and proliferative response to high-fat diet," Stem Cells, vol. 27, no. 10, pp. 2563-2570, 2009.

[3] S. B. Smith, H. Kawachi, C. B. Choi, C. W. Choi, G. Wu, and J. E. Sawyer, "Cellular regulation of bovine intramuscular adipose tissue development and composition," Journal of Animal Science, vol. 87, no. 14, pp. E72-82, 2009.

[4] P. Trayhurn, C. A. Drevon, and J. Eckel, "Secreted proteins from adipose tissue and skeletal muscle-adipokines, myokines and adipose/muscle cross-talk," Archives of Physiology and Biochemistry, vol. 117, no. 2, pp. 47-56, 2011.

[5] S. Galic, J. S. Oakhill, and G. R. Steinberg, "Adipose tissue as an endocrine organ," Molecular and Cellular Endocrinology, vol. 316, no. 2, pp. 129-139, 2010.

[6] B. Havekes and H. P. Sauerwein, "Adipocyte-myocyte crosstalk in skeletal muscle insulin resistance; is there a role for thyroid hormone?" Current Opinion in Clinical Nutrition \& Metabolic Care, vol. 13, no. 6, pp. 641-646, 2010.

[7] J.-P. Kovalik, D. Slentz, R. D. Stevens et al., "Metabolic remodeling of human skeletal myocytes by cocultured adipocytes depends on the lipolytic state of the system," Diabetes, vol. 60, no. 7, pp. 1882-1893, 2011.

[8] H. Sell, D. Dietze-Schroeder, and J. Eckel, "The adipocytemyocyte axis in insulin resistance," Trends in Endocrinology and Metabolism, vol. 17, no. 10, pp. 416-422, 2006.

[9] A. Mortazavi, B. A. Williams, K. McCue, L. Schaeffer, and B. Wold, "Mapping and quantifying mammalian transcriptomes by RNA-Seq", Nature Methods, vol. 5, no. 7, pp. 621-628, 2008.

[10] R. L. Baldwin, R. W. Li, C.-J. Li, J. M. Thomson, and B. J. Bequette, "Characterization of the longissimus lumborum transcriptome response to adding propionate to the diet of growing angus beef steers," Physiological Genomics, vol. 44, no. 10, pp. 543-550, 2012.

[11] H. He and X. Liu, "Characterization of transcriptional complexity during longissimus muscle development in bovines using high-throughput sequencing," PLOS ONE, vol. 8, no. 6, Article ID e64356, 2013.

[12] X. Sheng, H. Ni, Y. Liu, J. Li, L. Zhang, and Y. Guo, "RNA-seq analysis of bovine intramuscular, subcutaneous and perirenal adipose tissues," Molecular Biology Reports, vol. 41, no. 3, pp. 1631-1637, 2014.

[13] H.-J. Lee, M. Jang, H. Kim et al., "Comparative transcriptome analysis of adipose tissues reveals that ECM-receptor interaction is involved in the depot-specific adipogenesis in cattle," PLoS ONE, vol. 8, no. 6, Article ID e66267, 2013.

[14] P. Flicek, I. Ahmed, M. R. Amode et al., “Ensembl 2013," Nucleic Acids Research, vol. 41, no. 1, pp. D48-D55, 2013.

[15] S. Anders, D. J. McCarthy, Y. Chen et al., "Count-based differential expression analysis of RNA sequencing data using R and Bioconductor," Nature Protocols, vol. 8, no. 9, pp. 17651786, 2013.

[16] G. Dennis Jr., B. T. Sherman, D. A. Hosack et al., "DAVID: database for annotation, visualization, and integrated discovery," Genome Biology, vol. 4, no. 5, article P3, 2003.
[17] E. Albrecht, F. Teuscher, K. Ender, and J. Wegner, "Growthand breed-related changes of marbling characteristics in cattle," Journal of Animal Science, vol. 84, no. 5, pp. 1067-1075, 2006.

[18] J. R. Brethour, "Using serial ultrasound measures to generate models of marbling and backfat thickness changes in feedlot cattle," Journal of Animal Science, vol. 78, no. 8, pp. 2055-2061, 2000.

[19] A. L. J. Ferraz, A. Ojeda, M. López-Béjar et al., "Transcriptome architecture across tissues in the pig," BMC Genomics, vol. 9, article 173, 2008.

[20] W. Zhang, Q. D. Morris, R. Chang et al., "The functional landscape of mouse gene expression," Journal of Biology, vol. 3, no. 5 , article 21, 2004.

[21] R. Shyamsundar, Y. H. Kim, J. P. Higgins et al., "A DNA microarray survey of gene expression in normal human tissues," Genome Biology, vol. 6, no. 3, article R22, 2005.

[22] S. E. Buchalter, M. R. Crain, and R. Kreisberg, "Regulation of lactate metabolism in vivo," Diabetes/Metabolism Reviews, vol. 5, no. 4, pp. 379-391, 1989.

[23] C. E. Mondon, I. R. Jones, S. Azhar, C. B. Hollenbeck, and G. M. Reaven, "Lactate production and pyruvate dehydrogenase activity in fat and skeletal muscle from diabetic rats," Diabetes, vol. 41, no. 12, pp. 1547-1554, 1992.

[24] H.-X. Cui, R.-R. Liu, G.-P. Zhao, M.-Q. Zheng, J.-L. Chen, and J. Wen, "Identification of differentially expressed genes and pathways for intramuscular fat deposition in pectoralis major tissues of fast-and slow-growing chickens," BMC Genomics, vol. 13, no. 1, article 213, 2012.

[25] A. Teti, "Regulation of cellular functions by extracellular matrix," Journal of the American Society of Nephrology, vol. 2, no. 10, pp. S83-S87, 1992. 

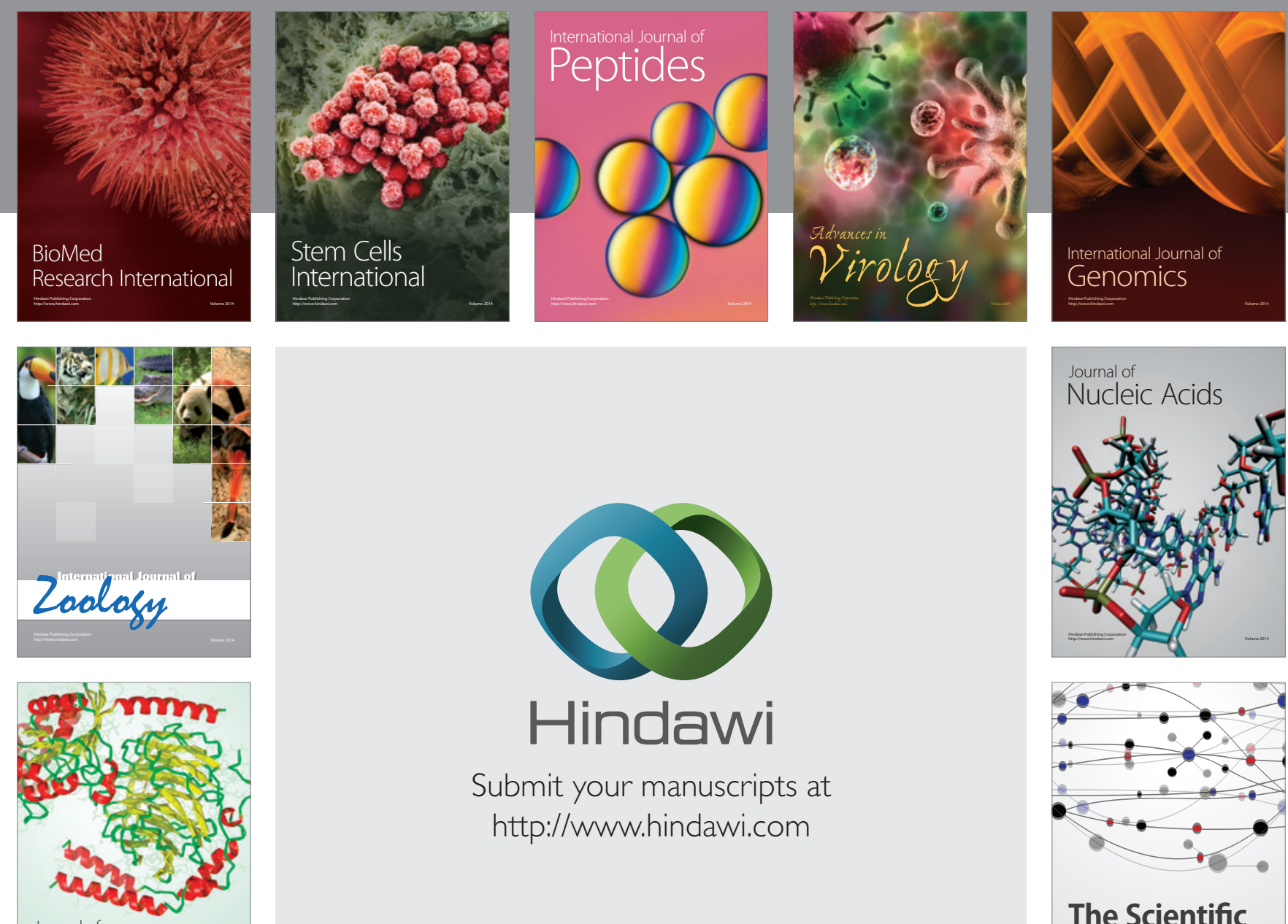

Submit your manuscripts at

http://www.hindawi.com

Journal of
Signal Transduction
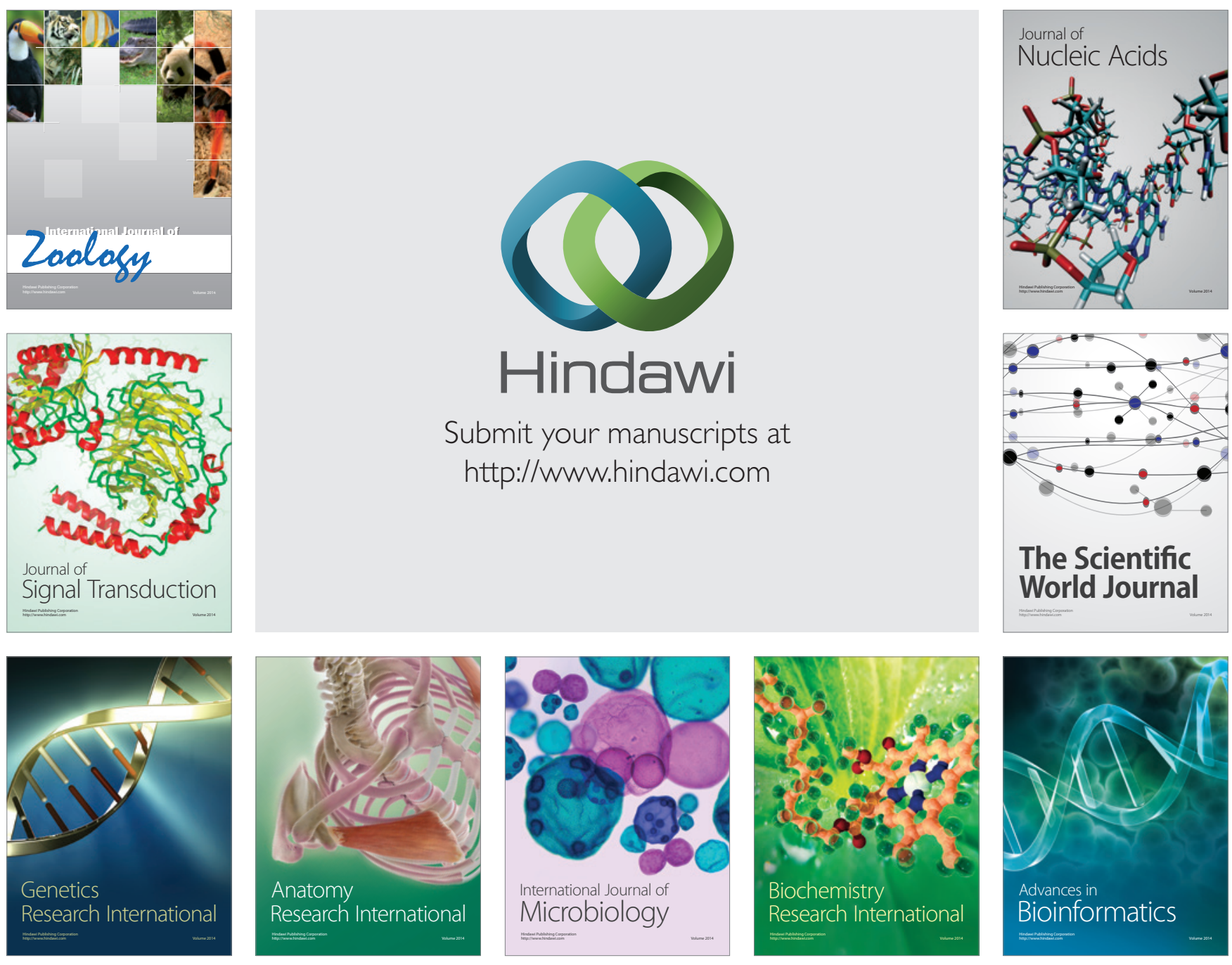

The Scientific World Journal
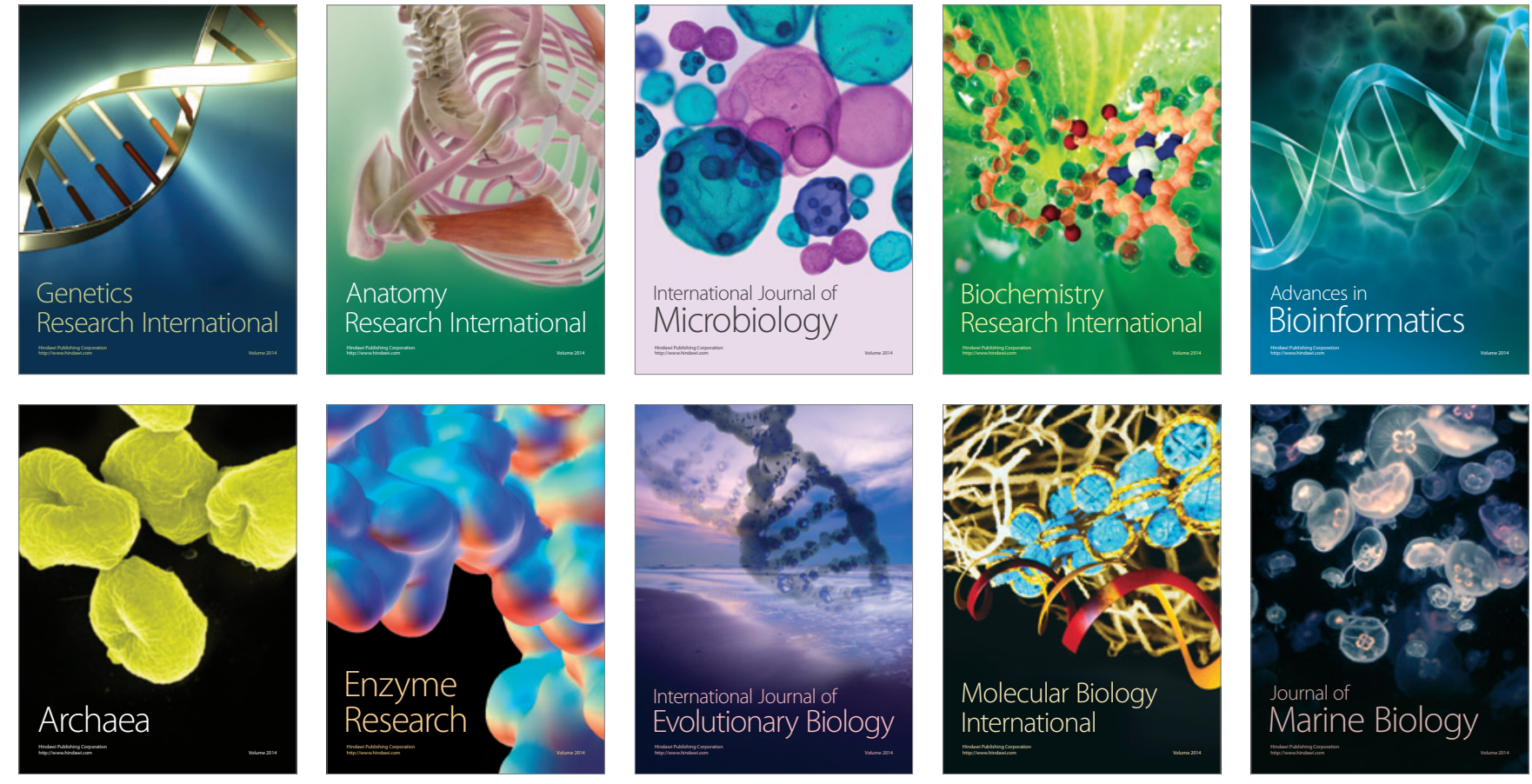